\title{
Gab2 facilitates epithelial-to-mesenchymal transition via the MEK/ERK/MMP signaling in colorectal cancer
}

\author{
Chenbo Ding ${ }^{1 \dagger}$, Junmin Luo ${ }^{1 \dagger}$, Longmei Li, Shanshan Li', Liwen Yang ${ }^{1}$, Hongfei Pan', Qianyi Liu', Huan Qin²,
} Chao Chen ${ }^{1}$ and Jihong Feng ${ }^{3^{*}}$

\begin{abstract}
Background: Grb2-associated binder 2 (Gab2), a scaffolding adaptor protein, has recently been implicated in cancer progression. However, the role of Gab2 in the progression and metastasis of colorectal cancer (CRC) remains unclear.

Methods: Gab2 expression was assessed in CRC patient specimens as well as in CRC cell lines. Recombinant lentivirus vector containing Gab2 gene and its small interfering RNAs were constructed and introduced into CRC cells. Cell migration and invasion ability were evaluated by transwell assays in vitro, and in vivo metastasis was performed on nude mice model. Moreover, the expression of Gab2 and epithelial-to-mesenchymal transition (EMT)-associated proteins (E-cadherin and vimentin) were assessed by western blot and QRT-PCR in CRC cells to evaluate the correlation between Gab2 and EMT. Finally, we evaluated the impact of Gab2 on the activation of its downstream signaling effectors, and furthermore the effects of these pathways on Gab2 induced-EMT were also detected.

Results: We confirmed that increased Gab2 expression correlated with higher tumor node metastasis stage and highly invasive CRC cell lines. Ectopic expression of Gab2 promoted metastasis of CRC cells, whereas silencing of Gab2 resulted in inhibited metastasis both in vitro and in vivo. Overexpression of Gab2 in CRC cells induced EMT, whereas knockdown of Gab2 had the opposite effect. Furthermore, upregulation of Gab2 expression obviously stimulated the activation of extracellular signal-regulated kinase-1/2 (ERK1/2), and increased the expression of matrix metalloproteinase-7 (MMP7) and matrix metalloproteinase-9 (MMP9) in CRC cells. Conversely, downregulation of Gab2 expression significantly decreased the activation of ERK1/2, and inhibited MMP7 and MMP9 expression. U0126, an inhibitor of mitogen-activated protein kinase (MEK), can reverse the effects of Gab2 on EMT.
\end{abstract}

Conclusions: Our work highlights that Gab2 induces EMT through the MEK/ERK/MMP pathway, which in turn promotes intestinal tumor metastasis.

Keywords: Gab2, Colorectal cancer, Metastasis, EMT

\section{Background}

Colorectal cancer (CRC) is the third most commonly carcinomas in males and the second in females throughout the world, with an estimated 1.4 million new cancer cases and nearly 0.7 million deaths each year [1]. The CRC incidence in the People's Republic of China has been increasing annually and it will continue to rise in the next years

\footnotetext{
* Correspondence: jh_f@163.com

${ }^{\dagger}$ Equal contributors

${ }^{3}$ Department of Oncology, the First Affiliated Hospital of Zunyi Medical College, Zunyi 563003, PR China

Full list of author information is available at the end of the article
}

[2]. Although the survival rate for patients with CRC has increased at early stages, as a result of improved detection and increased awareness, the long-term survival rate still remains very poor, mainly due to local recurrence and distant metastases formation [3, 4]. Metastasis, one of the six initial cancer hallmarks [5], which is a main reason of CRC-associated survival rate depressed [6]. Approximately $35 \%$ of patients with CRC have metastatic disease at diagnosis and more than one-third of patients will ultimately develop metastatic disease, however, the exact molecular mechanism underlying CRC metastasis is little known [7-9]. Improving understanding the key 
molecules in these processes, may provide novel insight for designing effective anti-cancer therapies.

Grb2-associated binder 2 (Gab2), a member of the DOS/ Gab family of scaffolding adapters, has been reported to play important roles in the progression and metastasis of human cancers, particularly in breast and ovarian cancers and melanoma [10]. Although Gab2 has a modest impact on mammary tumor initiation/growth, Gab2 deletion leads to impaired ERK activity and attenuated mammary tumor metastasis [11]. Gab2 triggers epithelial-to-mesenchymal transition (EMT) and promotes the migration and invasion of ovarian cancer cells through activation of the phosphatidylinositol 3-kinase (PI3K) pathway [12]. In melanoma, Gab2 expression promotes the migration, invasion and metastasis of tumor cells via activation of the PI3K/ AKT signaling [13]. These data indicates that Gab2 and Gab2-mediated signaling pathways are involved in the metastasis of human tumor cells.

However, whether Gab2 has any role in the metastasis of $\mathrm{CRC}$ and its underlying mechanism remains unknown. In this study, the effects of Gab2 on CRC metastasis as well as its relative molecular mechanism were investigated using in vitro CRC cell lines and in vivo mouse model systems. We demonstrate that Gab2 induces EMT by the MEK/ERK/ MMP pathway, which in turn promotes CRC cells metastasis. The current findings suggest that Gab2 plays a vital role in regulating CRC metastasis and may serve as a potential target for diagnosis and therapy in CRC.

\section{Methods}

\section{Tissue samples}

Samples from 35 CRC patients who had undergone proctocolectomy with lymph node (LN) dissection for CRC at the Department of gastrointestinal Surgery, the First Affiliated Hospital of Zunyi Medical College (Zun'yi, China) between May 2015 and July 2015 were included in the study. Patients did not receive neoadjuvant therapy. After surgical resection, the resected specimens were histologically examined by hematoxylin and eosin (HE)-staining and were immediately put into liquid nitrogen until further use. Total RNA from the frozen tissues was isolated with Trizol (Invitrogen, USA) according to the manufacturer's instructions. All patients provided written informed consent before surgery, and our study were approved by the Ethics Committee of the First Affiliated Hospital of Zunyi Medical College according to the 1975 Declaration of Helsinki.

\section{Cell lines and culture conditions}

The human CRC cell lines HT29, SW480, SW620 and LOVO, and a normal human intestinal epithelial cell line FHC were obtained from the American Type Culture Collection (ATCC, Manassas, USA), and authenticated according to the ATCC recommendations. SW480 and SW620 cells were cultured in Leibovitz's L-15 medium (GIBCO Laboratories, Grand Island, NY, USA) supplemented with $10 \%$ fetal bovine serum (FBS) (HyClone, Logan, UT, USA), $100 \mathrm{U} / \mathrm{ml}$ penicillin and $100 \mu \mathrm{g} / \mathrm{ml}$ streptomycin. HT29 cells were cultured in McCoy's medium (GIBCO) supplemented with $10 \%$ FBS and antibiotics. LOVO and FHC were maintained in RPMI-1640 medium (HyClone) supplemented with $10 \%$ FBS and antibiotics. All the cells were cultured at $37{ }^{\circ} \mathrm{C}$ in a humidified incubator of $5 \% \mathrm{CO}_{2}$.

\section{RNA extraction and real-time PCR}

Total RNA was extracted using Trizol (Invitrogen, USA) according to the manufacturer's instructions. The obtained RNA was first reversely transcribed into cDNA by using RT reagent Kit (TakaRa, Japan). Quantitative reverse transcription-PCR (qRT-PCR) analysis was performed as previously described [14]. The sequences of primers in this section are the followings: (1) Gab2: 5'-GTGGGGGATCTGAATGTTTTTATG-3' (forward) and 5' -GCCCCAGGGTAGAATGAAACG-3' (reverse); (2) E-cadherin: 5'-GCTCGGCCTGAAGTG ACTCG-3' (forward) and 5'-CCGCTTCCTTCATAG TCAAACAC-3' (reverse); (3) Vimentin: 5'-CCAGGC AAAGCAGGAGTCCAC-3' (forward) and 5'-GCTT CCTGTAGGTGGCAATCTC-3' (reverse); (4) GAPDH: 5'-GAAGGTGAAGGTCGGAGTC-3' (forward) and 5'-GAAGATGGTGATGGGATTTC-3' (reverse). GAP DH was used as an internal control.

\section{Western blot analysis}

Western blot analysis was performed as previously described [14]. The following commercial antibodies were used in this study: Gab2 (OriGene Technologies, USA), E-cadherin, vimentin, MMP7 and MMP9 (Abcam, UK), phospho-AKT, total AKT, phospho-ERK1/2 and total ERK1/2 (Invitrogen, USA), GAPDH and $\beta$-actin (Immunology Consultants Laboratory, USA).

\section{Immunohistochemical staining}

Immunohistochemical assay was performed on paraformaldehyde-fixed paraffin sections as previous reported [14]. The Gab2 (OriGene Technologies, USA) primary antibody was used at a 1:150 dilution in the immunohistochemistry assays. The immunostaining intensity and average percentage of positive cells were evaluated as previous reported [14]. According to the chromatosis intensity, no staining, light yellow, buffy and brown are scored $0,1,2$, and 3, respectively. And the percentage of positive cells was categorized as the following grades: 0 (less than $5 \%$ ), 1 (5-25\%), 2 (26-50\%), and 3 (>51\%) accordingly. By multiplying the staining intensity and the percentage of positive cells, the final weighed expression score was ranged from 0 to 9 . The sum of the staining intensity score and the percentage score was used to define 
the Gab2 protein expression levels: 0-2, low expression and 3-9, high expression.

\section{Lentivirus production and construct design}

Retroviral expression vector CMV-puro and CMV-puro containing Gab2 gene were designed and provided by Cyagen Biosciences Inc. (Guangzhou, China). On the basis of the Gab2 sequence, one short hairpin RNA was designed using the small interfering RNAs (siRNAs) Target Finder (InvivoGene, San Diego, CA, USA). The effective Gab2 siRNA sequence is 5'- GCACCAATTCTG AAGACAA - $3^{\prime}$, and the control-siRNA sequence is 5' -TTCTCCGAACGTGTCACGT-3'. Lentiviral vectors encoding siRNAs and the control-siRNA sequence were designed and provided by Genechem lnc. (Shanghai, China).

\section{Migration and invasion assay}

Migration and invasion assays were using Transwell system (24-well, $8 \mu \mathrm{m}$ pore size with polycarbonate membrane; Corning Costar, Lowell, MA, USA). Six hundred microlitres of the medium with $15 \%$ FBS was added to the lower well of each chamber and $5 \times 10^{4}$ cells/well resuspended in $200 \mu \mathrm{l}$ serum-free medium were added to the upper inserts. After $48 \mathrm{~h}$ incubation, the cells that had migrated through the filter into the lower wells were stained with $0.1 \%$ crystal violet and counted using fluorescence microscopy. The invasion assay was performed in the same way as the migration assay except that the membrane was coated with Matrigel (BD Biosciences, SanJose, CA, USA). Three independent experiments were performed with triplicate wells. DMSO (vehicle) or U0126 was added to both the top and bottom of transwell.

\section{In vivo metastasis development assay}

To produce experimental lung and liver metastasis, SW480-NC, SW480-Gab2, SW620-si-Ctrl and SW620Gab2si cells $\left(1 \times 10^{6}\right.$ cells $)$ were injected into the lateral tail veins of 5-6 weeks-old BALB/c nu/nu female mice (six mice per group). After 4 weeks, all the mice were killed under anesthesia. The lungs and livers were collected and fixed in $10 \%$ formalin. For tissue morphology evaluation, HE-staining was performed on sections from embedded samples. All animal experiments were performed with the approval of Zunyi Medical College Animal Care and Use Committee.

\section{Statistical analysis}

All values were represented as the mean \pm SEM from at least three independent experiments. Pearson's $\chi^{2}$-test was used for clinical correlative studies. Student's t-test for two groups or one-way analysis of variance (ANOVA) for

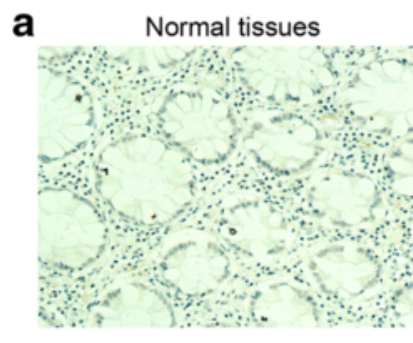

b

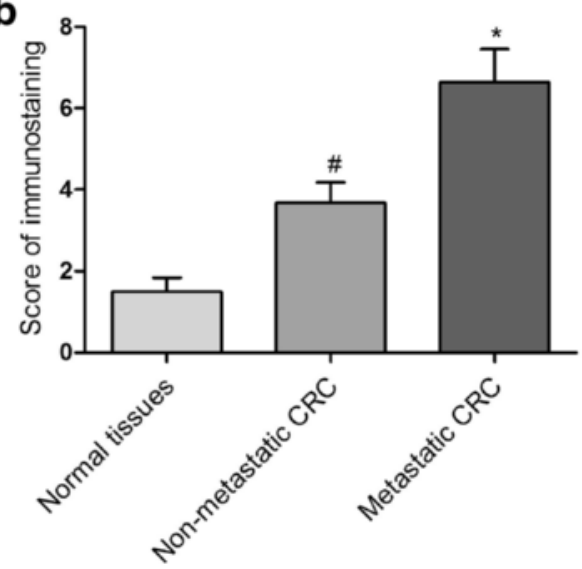

Non-metastatic CRC

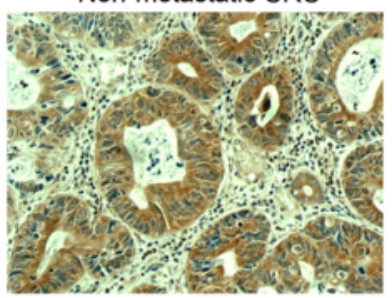

Metastatic CRC

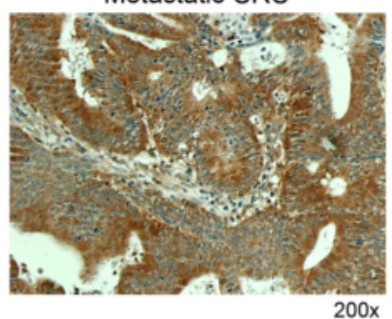

C

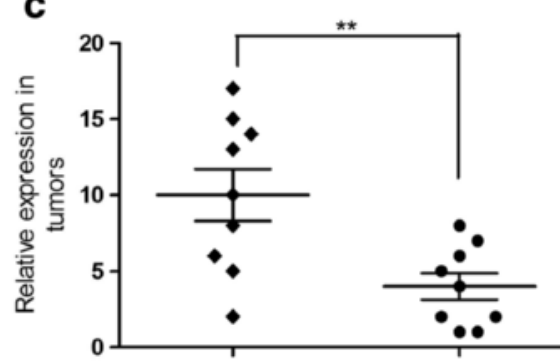

Fig. 1 Gab2 is significantly upregulated in LN metastasis-positive CRC tissues. a Immunohistochemistry analysis of Gab2 expression in 35 paired CRC tissues. b Results of immunohistochemical staining were evaluated by the staining scores. ${ }^{*} P<0.05$ vs non-metastatic CRC or normal tissues. ${ }^{\#} P<0.05$ vs normal tissues. c qRT-PCR analysis of Gab2 expression in human CRC primary tumors, LN-positive $(n=9)$ or - negative $(n=9)$. Matching normal colorectal tissue sample from the same patient was used for normalization 
three or more groups were performed to evaluate the statistical significance. Differences were considered significant at $P$ values less than 0.05 .

\section{Results}

Gab2 is significantly upregulated in LN metastasis-positive

\section{CRC tissues}

Our previous study has shown that Gab2 is overexpressed in CRC tissues, and this overexpression is significantly correlated with lymph node (LN) metastasis [14]. In this study, we also assessed Gab2 expression in a tissue microarray of 35 CRC patients (Additional file 1: Table S1). The results of immunohistochemical staining showed that Gab2 was significantly upregulated in primary sites of metastatic CRC compared with either non-metastatic CRC or normal tissues (Fig. 1a, b). To investigate the correlation between Gab2 overexpression and CRC metastasis, we detected Gab2 expression in 9 pairs of LN metastasispositive (LN-positive group) and $\mathrm{LN}$ metastasis-negative (LN-negative group) primary CRC specimens. Real-time PCR analysis showed that Gab2 mRNA level was obviously higher in the LN-positive group than in the LN-negative

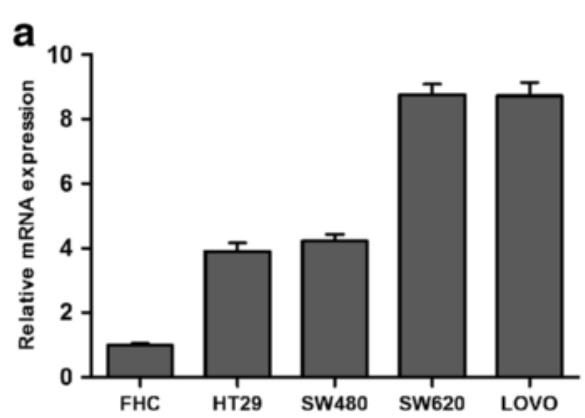

b

C

SW480-NC SW480-Gab2
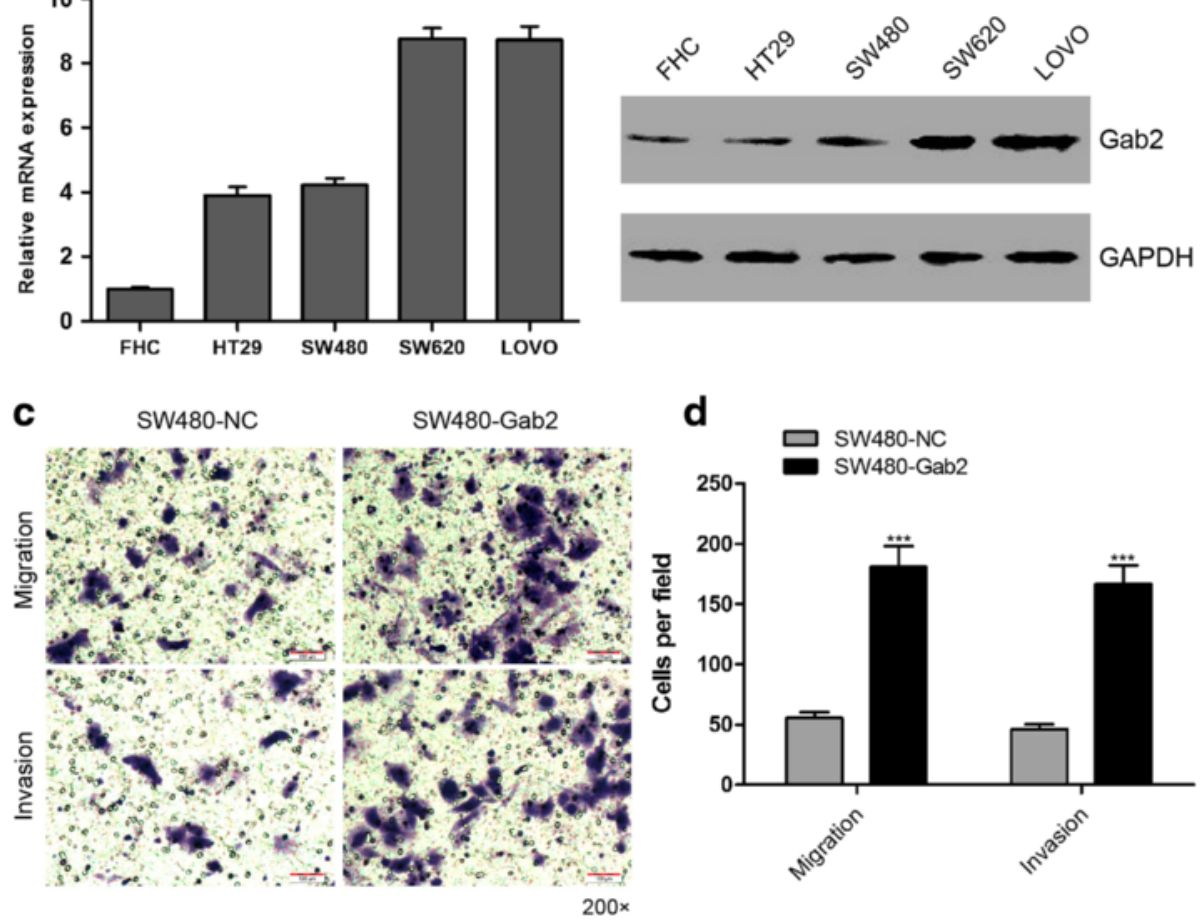

e SW620-si-Ctrl SW620-Gab2si

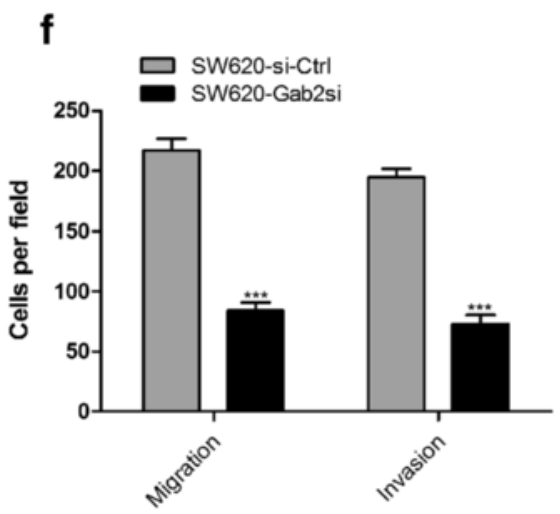

Fig. 2 Gab2 promotes CRC cells migration and invasion in vitro. a Expression of Gab2 in four human CRC cell lines relative to the normal human intestinal epithelial cell line FHC was detected by qRT-PCR. b Western blot analysis of Gab2 expression in different CRC cell lines. c Migration and invasion assay of SW480-NC and SW480-Gab2 cells. $\mathbf{d}$ Migration and invasion of SW480-NC and SW480-Gab2 cells were quantitatively analyzed. Columns are the average of three independent experiments \pm SEM. e and $\mathbf{f}$ Migration and invasion assay of SW620-si-Ctrl and SW620-Gab2si cells (two clones, $\mathbf{c}$ and $\mathbf{d}$ ). ${ }^{* * *} P<0.001$ 
group (Fig. 1c). Taken together, these results suggest that the expression of Gab2 is positively correlated with the metastasis of CRC.

\section{Gab2 accelerates CRC cell migration and invasion in vitro}

To determine whether Gab2 expression associates with the metastatic potential of CRC cells, we detected the expression of Gab2 in four human CRC cell lines (HT29, SW480, SW620 and LOVO) and in a normal human intestinal epithelial cell line FHC. The levels of Gab2 expression were obviously increased in SW620 and LOVO cells, which have highly metastatic abilities, compared with either the poorly metastatic cell lines HT29 and SW480 or the normal human intestinal epithelial cell line FHC (Fig. 2a, b). Considered that SW480 and SW620 cells were isolated from a same patient [15], these cells therefore have the same genetic background but different metastatic potential [16].

To explore whether Gab2 affects the metastatic phenotype of CRC cells, SW480 cells were infected with lentiviral vectors containing Gab2 gene or a control lentivirus and stable clones were established (SW480-Gab2 and SW480$\mathrm{NC}$, respectively). Gab2-specific small interfering RNAs (siRNAs) or its corresponding control siRNA were introduced into SW620 cells and stable clones were established
(SW620-Gab2si and SW620-si-Ctrl, respectively). In order to determine the generality of the impact of Gab2 regulation in cell metastasis, we adopted transwell assays. As results, upregulation of Gab2 expression in SW480 cells significantly enhanced cell migration and invasion (Fig. 2c, d). Conversely, downregulation of Gab2 in SW620 cells markedly reduced cell migration and invasion (Fig. 2e, f). These data indicates that Gab2 promotes metastasis of CRC cells in culture.

\section{Gab2 induces metastasis in a xenograft model}

Next, we studied the effect of Gab2 on tumor metastasis in vivo. SW480-NC, SW480-Gab2, SW620-si-Ctrl and SW620-Gab2si cells were transplanted into the nude mice via tail vein injection. After 4 weeks, mice were anesthetized, and their lungs and livers were dissected and HEstaining was performed to evaluate tissue morphology. Histologic analyses confirmed that the incidence of lung and liver metastases was obviously enhanced in mice injected with SW480-Gab2 cells and significantly decreased in mice injected with SW620-Gab2si cells, compared with the corresponding control groups (Fig. 3a). Two out of six mice injected with SW480-NC cells had small lung metastatic nodules, whereas four out of six mice in the SW480-Gab2 group ended up with heavy lung
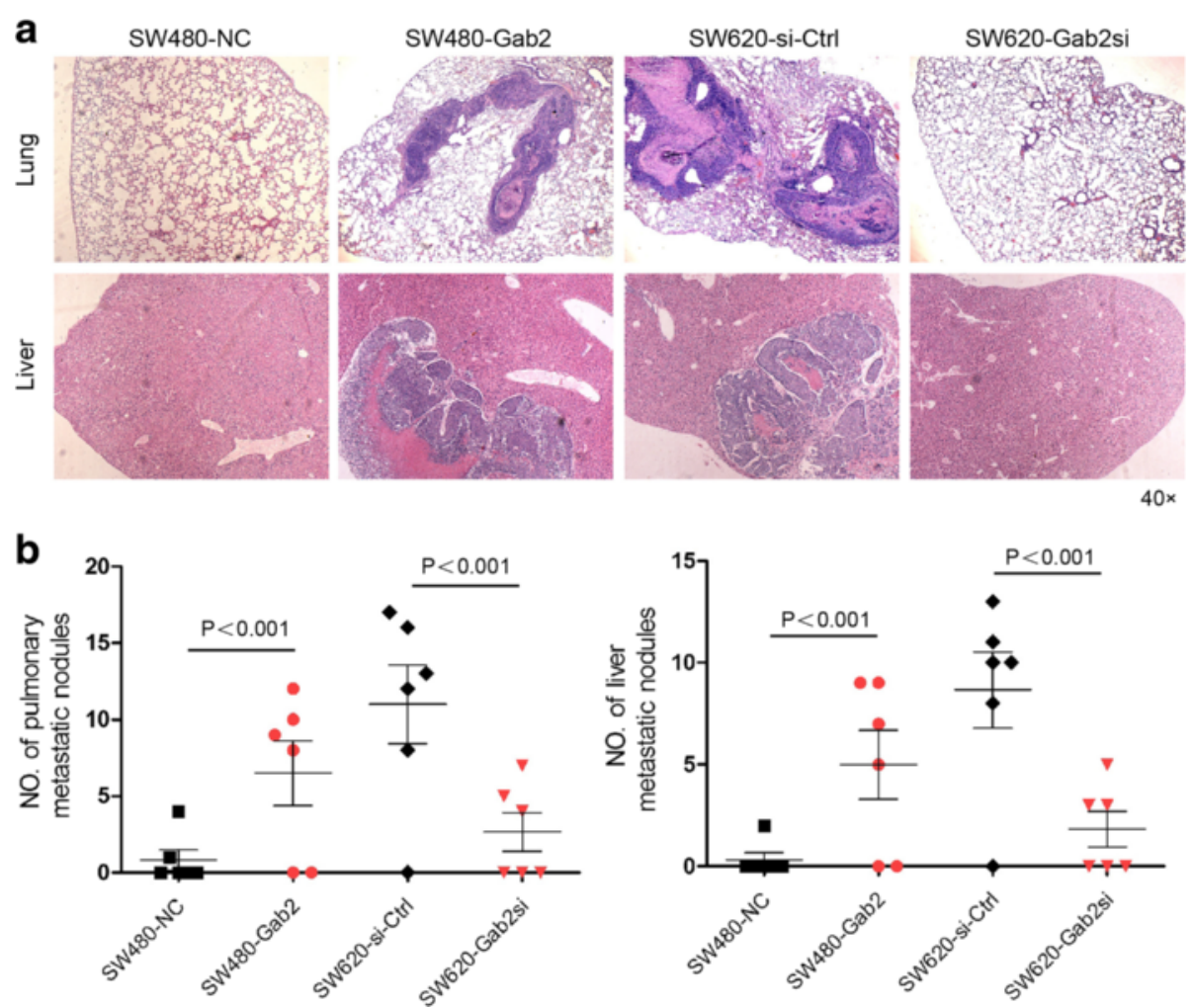

Fig. 3 Gab2 enhances CRC cells metastasis in a xenograft model. a Images showing representative HE-staining of lungs and livers isolated from mice that received tail vein injection of SW480-NC, SW480-Gab2, SW620-si-Ctrl and SW620-Gab2si cells. Each group contains six mice. $\mathbf{b}$ The numbers of pulmonary and liver metastatic nodules were counted and analyzed with Student's t-test 
metastasis. Conversely, silencing of Gab2 expression in SW620 cells had small lung metastatic nodules when compared with the control group. Moreover, the counts of lung and liver metastatic nodules exhibited significantly increased in the SW480-Gab2 group and significantly decreased in SW620-Gab2si group, compared with the corresponding control groups (Fig. 3b). These results suggest that Gab2 can enhance CRC cells metastasis in vivo.

\section{Gab2 regulates EMT in CRC cell}

The EMT is a powerful process in tumor invasion and metastasis. During the EMT process, the molecular reprogramming and phenotypic changes characterized by a transition from polarized immotile epithelial cells to motile mesenchymal cells, thus leading to increased motility and invasion [17]. Moreover, the transition is characterized by a decrease in the expression of epithelial markers (such as E-cadherin) as well as an increase in the expression of mesenchymal markers (such as vimentin) $[18,19]$.

By comparing the morphology of the cell models described above under a light microscope, we found that elevated expression of Gab2 in SW480 cells induced the conversion of polarized epithelial cells to spindle-shaped, fibroblast-like mesenchymal cells with decreased cell-cell contact. Conversely, silencing of Gab2 in SW620 cells exhibited an increase in cell-cell adhesion and an epithelioid morphology (Fig. 4a). To confirm that Gab2 induced EMT to promote CRC metastasis, we assessed the expression of EMT-markers in these cell models. As results, a decrease in the expression of E-cadherin and an increase in the expression of vimentin were observed in SW480-Gab2 cells, compared with the control cells (Fig. 4b, c). By contrast, a marked decrease in the expression of vimentin and a significant increase in the expression of E-cadherin were observed in SW620-Gab2si cells, compared with the control cells (Fig. 4b, d). These results suggest that Gab2 is critical for the acquisition of EMT characteristics and may contribute to the EMT-induced invasive phenotype in CRC cells.

\section{Gab2 positively regulates ERK signaling in CRC cell}

Recent studies have shown that the expression of several MMPs was enhanced in many types of cancer [20-22]. In these MMPs, it has been demonstrated that MMP7 and MMP9 were involved in the progression and metastasis of CRC [23-25]. To investigate the possible mechanism of Gab2 participating in cell metastasis of colorectal carcinoma, we examined its downstream effectors, AKT and ERK. Overexpression of Gab2 in SW480 cells significantly increased phosphorylation of ERK1/2, whereas knockdown

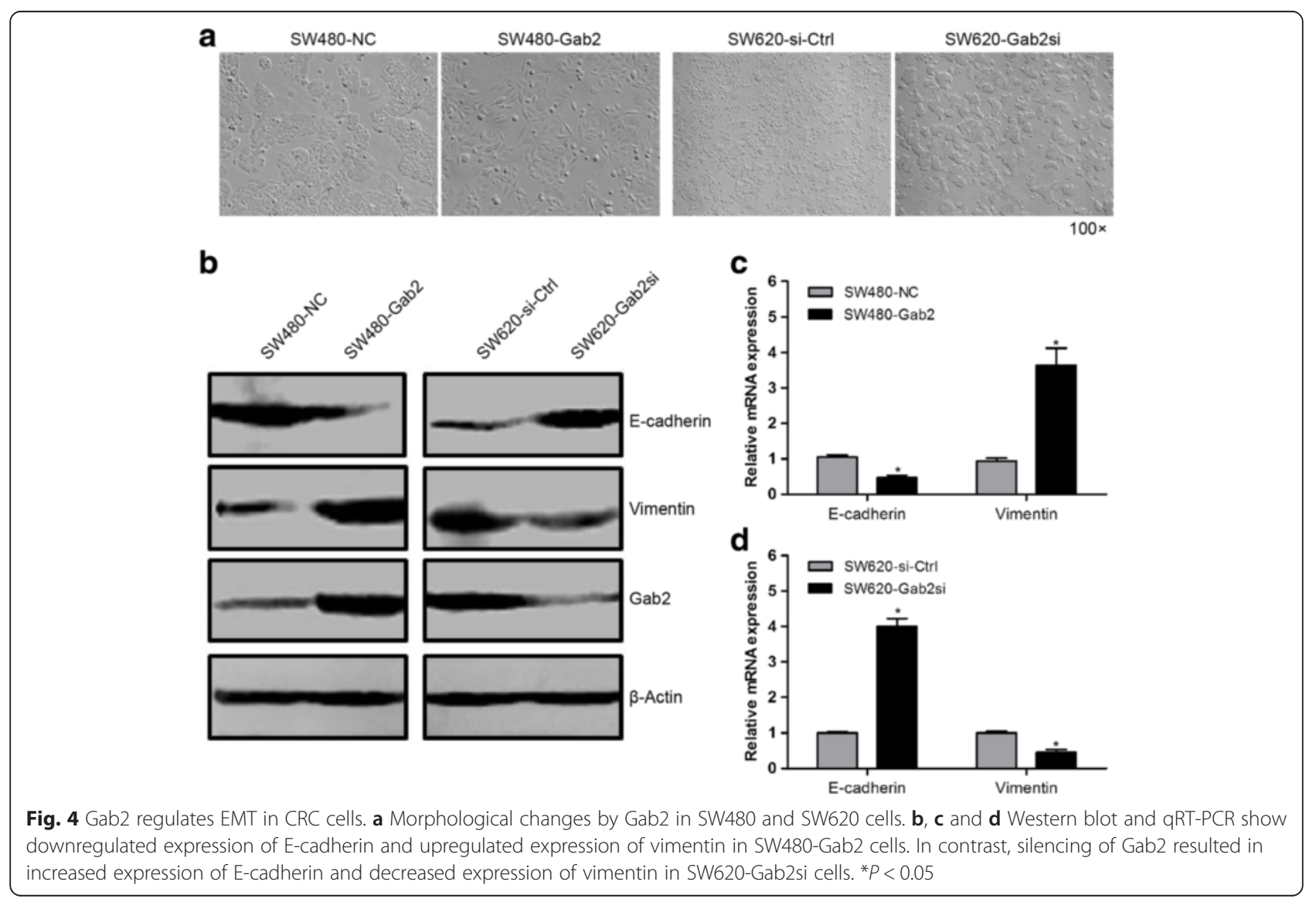


of Gab2 in SW620 cells obviously reduced the activation of ERK1/2 (Fig. 5). Not as expected, the phosphorylation of AKT did not show marked increased or decreased in these cells (Fig. 5). Moreover, we also detected the expression of MMP7 and MMP9 in these cells, and found that increased MMP7 and MMP9 expression in SW480-Gab2 cells compared with the control cells, and decreased MMP7 and MMP9 expression in SW620-Gab2si cells compared with the control cells (Fig. 5). These data indicates that Gab2 may enhance CRC cells metastasis through the ERK/MMP pathway.

Pharmacological inhibitor of MEK inhibits Gab2-induced EMT To test the hypothesis that the commissioning of EMT by Gab2 is required for the activation of MEK/ERK signaling in colorectal carcinoma, we explore whether U0126, an effective MEK inhibitor, can inhibit Gab2-induced EMT and cell migration and invasion in CRC. Western blot analyses confirmed that the expression of E-cadherin was partially restored and the expression of vimentin, MMP7 and MMP9 was reduced upon using U0126 in SW480-Gab2 cells (Fig. 6a). Accordingly, migration and invasion of SW480-Gab2 cells were analyzed in the absence or presence of U0126 using transwell migration and invasion assays. As results, migration and invasion of SW480-Gab2 cells were significantly reduced in the presence of U0126 (Fig. 6b, c). These data indicates that Gab2-induced EMT and cell metastasis is dependent on MEK/ERK/MMP signaling in CRC.

\section{Discussion}

It has been widely recognized that MEK/ERK signaling is participated in the progression and metastasis of CRC [26-28]. In addition, expression of a constitutively active mutant of MEK1 or MEK2 in rodent normal intestinal epithelial cells (IECs) is sufficient to induce growth, EMT and formation of invasive metastatic tumors in nude mice $[29,30]$. In this study, we have shown that Gab2 promotes cell migration and invasion of CRC through activation of the MEK/ERK pathway, further showing the importance of ERK signaling for CRC metastasis. Additionally, we provide evidence supporting the involvement of Gab2 in the regulation of EMT in cancer.

Gab2 has recently been proposed to be a critical molecule in the regulation of cancer metastasis [10], although the exact mechanism of Gab2 in metastasis remains unclear. We found that overexpression of Gab2 enhanced CRC cell migration and invasive properties. Conversely, knockdown of Gab2 had the opposite effect. In accordance with our study, Gab2 was reported to be one of the
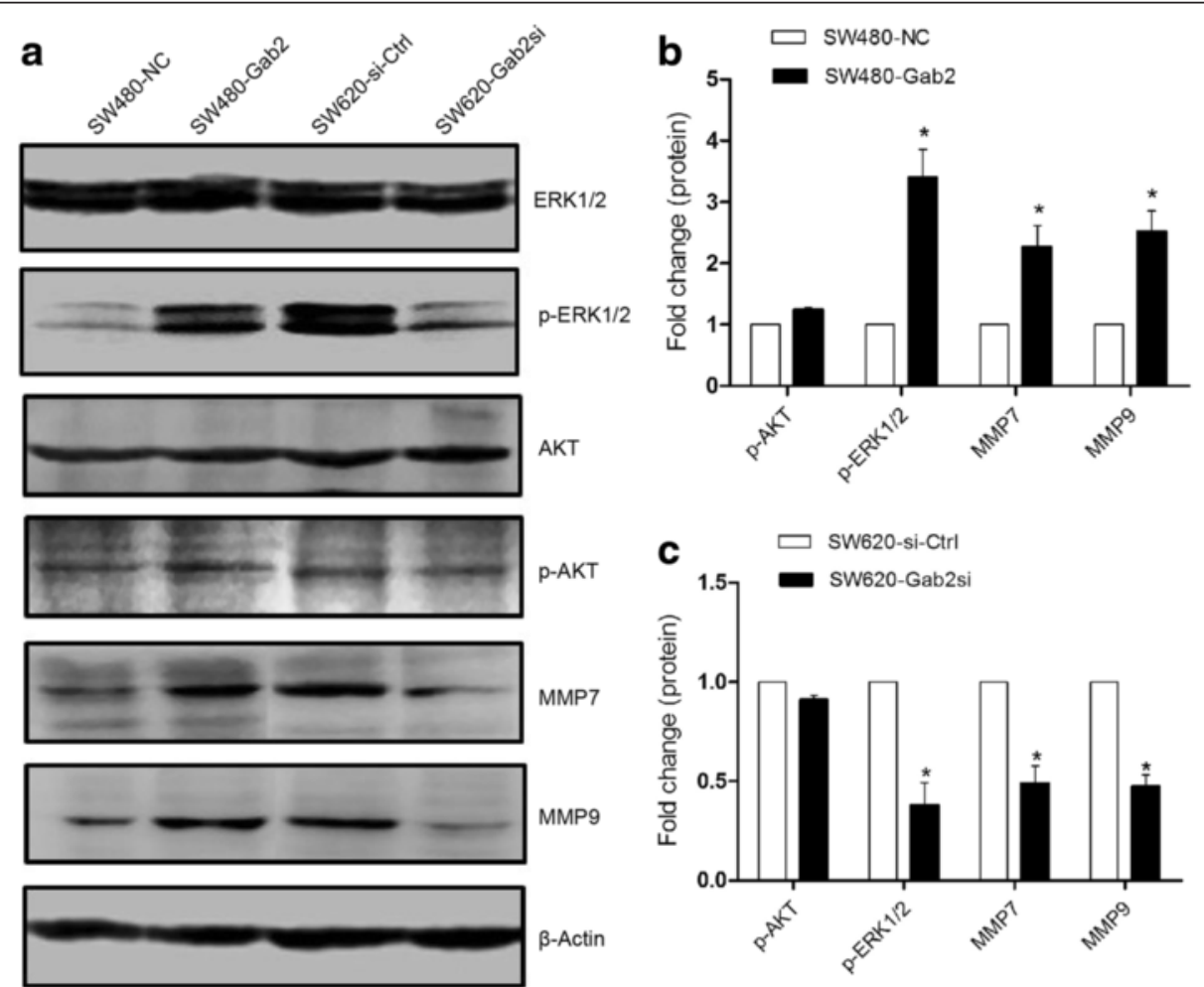

Fig. 5 Gab2 expression regulates ERK signaling and increases MMP7 and MMP9 expression in CRC cells. a Gab2 overexpression enhances MMP7 and MMP9 expression and activates ERK1/2, whereas knockdown of Gab2 inhibits MMP7 and MMP9 expression and reduces ERK1/2 activation. b and $\mathbf{c}$ The levels of phosphor-AKT, phosphor-ERK1/2, MMP7 and MMP9 were calculated. The data are representative of at least three different experiments \pm SEM. ${ }^{*} P<0.05$ 

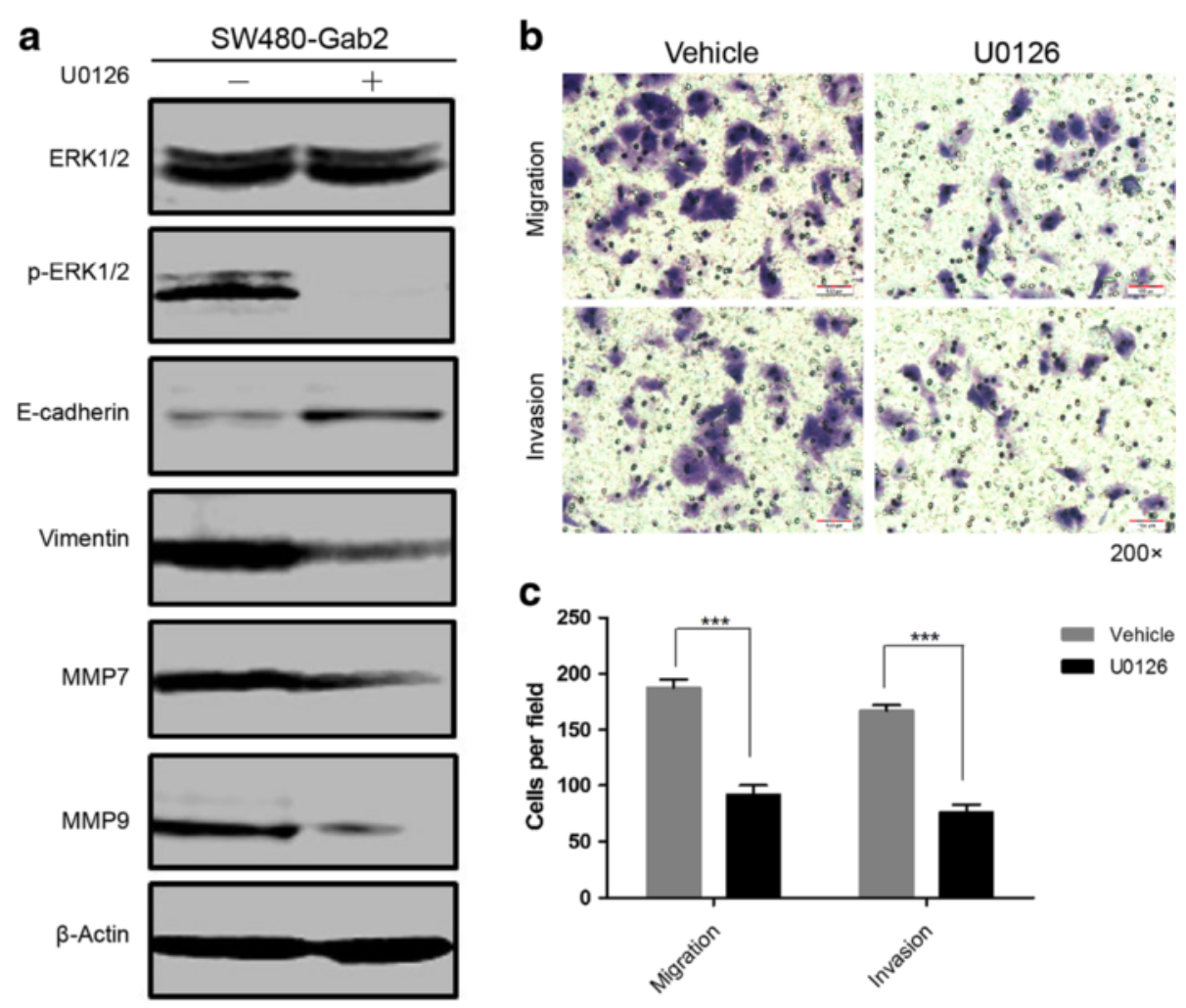

Fig. 6 Inhibitor of MEK reduces Gab2-induced EMT, cell migration and invasion. a SW480-Gab2 cells were treated or not with $20 \mu M$ U0126 during $16 \mathrm{~h}$ after which proteins were analyzed by western blot with specific antibodies against E-cadherin, vimentin, MMP7, MMP9, phosphorylated ERK1/2 and total ERK1/2. $\mathbf{b}$ and $\mathbf{c}$ SW480-Gab2 cells were subjected to transwell migration and invasion assays in the absence (vehicle), or presence of $20 \mu \mathrm{M}$ U0126. ${ }^{* * *} P<0.001$

molecules essential for both ovarian cancer and melanoma $[12,13]$. Similarly, another study indicated that the level of Gab2 expression was significantly associated with metastatic progression of breast cancer [31]. Moreover, efficient ErbB2-driven mammary tumorigenesis and metastatic spread requires Gab2 expression [32]. Our results shed new light on the role of Gab2 in the promotion of cancer metastasis.

It has been reported that Gab2 regulates cytoskeletal organization and migration of mammary epithelial cells by modulating RhoA activation [33]. Interestingly, a recent study has shown that miR125a-5p could inhibit migration and invasion of glioma cells by mediating Gab2 to affect cytoskeleton rearrangement and MMPs expression [34]. These studies suggest that Gab2 might promote cancer metastasis by regulating the EMT. In this study, we demonstrated that upregulation of Gab2 expression induced the conversion of polarized epithelial cells to spindleshaped, fibroblast-like mesenchymal cells with decreased cell-cell contact, enhanced invasion and migration in CRC cells, upregulated vimentin, MMP7 and MMP9 and downregulated E-cadherin. These findings provided new evidence supporting the involvement of Gab2 in driving cancer cells metastasis through the regulation of EMT.
Of further interest, we examined the possible pathway of Gab2 participating in cell metastasis of colorectal carcinoma and found that increased ERK1/2 phosphorylation in Gab2-upregulated CRC cells. Unfortunately, we did not detect any significant association between AKT phosphorylation and the expression of Gab2 in CRC cells. In addition, pharmacological inhibitor of MEK could reverse the effects of Gab2 on CRC cells migration and invasion, and partially restore E-cadherin expression, suggesting that Gab2-induced EMT is dependent on MEK/ERK signaling in CRC. Notably, activating genetic mutations in the PI3K/AKT and the MEK/ERK pathways have been implicated in CRC. Gab2 has been recognized as critical activators of the PI3K/AKT and/or the SHP2/ERK pathways in several cellular systems [10]. In theory, the level of Gab2 should affect AKT and ERK phosphorylation in CRC cells. However, SW620 cells transformed by downregulation of Gab2 did not show marked reduction of AKT phosphorylation in comparison with control cells. Similarly, at higher concentration ratios of PI3K/SHP2 in VEGF-stimulated endothelial cells, the lipid kinase is not limiting and AKT is phosphorylated to the same extent regardless of Gab2 knockdown, indicated that sufficient PI3K is activated for maximal AKT phosphorylation [35]. 
One plausible explanation is that the expression of Gab2 cannot markedly affect the concentration ratios of PI3K in these cells. Additionally, other Gab2-mediated oncogenic pathways need to be analyzed in order to clearly determine whether there is a link between Gab2 expression and EMT startup.

In summary, our study demonstrates that Gab2 could induce EMT and promote CRC cell metastasis through the MEK/ERK/MMP signaling. However, further studies are needed to pinpoint the target gene of Gab2, which can act as Gab2 promoters/suppressors regulated CRC metastasis. The present study provides a novel fundamental insight into how Gab2 promotes metastasis in colorectal carcinoma.

\section{Conclusions}

Our study provides a better understanding on both the molecular mechanism and functional role of Gab2 in human CRC. Our current work revealed that Gab2 exerted its role as oncogene in CRC by facilitating cancer cell migration and invasion. Notably, Gab2 can upregulate MMP7 and MMP9 expression via MEK/ERK signaling pathway in CRC. Our study identified that Gab2 is a novel regulator of EMT through Gab2-medated MEK/ERK/MMP signaling, indicating its potential therapeutic value for reducing CRC metastasis.

\section{Additional file}

Additional file 1: Table S1. Clinicopathologic factors and Gab2

expression in 35 CRC patients. (DOCX $22 \mathrm{~kb}$ )

\section{Competing interests}

The authors declare that they have no competing interests.

\section{Authors' contributions}

CBD conceived and designed the experiments. CBD, LML and SSL performed the experiments. LWY, HFP, QYL, HQ and CC analyzed the data. LML helped in sample collection. CBD wrote the paper. JHF and JML supervised the whole experimental work. All authors read and approved the final manuscript.

\section{Acknowledgements}

This study was supported by grants from the National Science Foundation of China (No. 81560407), Key Project on Social Development by Department of Science and Technology of Guizhou Province (No. 2015SY3046), the Joint Foundation of Guizhou Province (No. 2015LH7527), the Science and Technology Foundation of Guizhou Province (No. 2013J2312) and the Doctoral Scientific Research Foundation of Affiliated Hospital of Zunyi Medical College (No. 2014-7).

\section{Author details}

${ }^{1}$ Department of Immunology, Zunyi Medical College, Immunology Innovation Base of Postgraduate Education in Guizhou Province, Zunyi 563003, PR China. ²Department of Microbiology, Zunyi Medical College, Zunyi 563003, PR China. ${ }^{3}$ Department of Oncology, the First Affiliated Hospital of Zunyi Medical College, Zunyi 563003, PR China.

Received: 1 November 2015 Accepted: 29 December 2015 Published online: 12 January 2016

\section{References}

1. Torre LA, Bray F, Siegel RL, Ferlay J, Lortet-Tieulent J, Jemal A. Global cancer statistics, 2012. CA Cancer J Clin. 2015;65(2):87-108.

2. Dai Z, Zheng RS, Zou XN, Zhang SW, Zeng HM, Li N, et al. [Analysis and prediction of colorectal cancer incidence trend in China]. Zhonghua yu fang yi xue za zhi. Chin J Prev Med. 2012;46(7):598-603.

3. Chua YJ, Zalcberg JR. Progress and challenges in the adjuvant treatment of stage II and III colon cancers. Expert Rev Anticancer Ther. 2008;8(4):595-604.

4. Speetjens FM, Zeestraten EC, Kuppen PJ, Melief CJ, van der Burg SH. Colorectal cancer vaccines in clinical trials. Expert Rev Vaccines. 2011;10(6): 899-921.

5. Hanahan D, Weinberg RA. Hallmarks of cancer: the next generation. Cell. 2011;144(5):646-74.

6. Christofori G. New signals from the invasive front. Nature. 2006;441(7092): 444-50

7. Field K, Lipton L. Metastatic colorectal cancer-past, progress and future. World J Gastroenterol. 2007;13(28):3806-15.

8. Jemal A, Siegel R, Ward E, Murray T, Xu J, Smigal C, et al. Cancer statistics, 2006. CA Cancer J Clin. 2006:56(2):106-30.

9. Zhao L, Wang H, Liu C, Liu Y, Wang X, Wang S, et al. Promotion of colorectal cancer growth and metastasis by the LIM and SH3 domain protein 1. Gut. 2010;59(9):1226-35.

10. Ding CB, Yu WN, Feng JH, Luo JM. Structure and function of Gab2 and its role in cancer (Review). Mol Med Rep. 2015;12(3):4007-14.

11. Ke $Y$, Wu D, Princen F, Nguyen T, Pang Y, Lesperance J, et al. Role of Gab2 in mammary tumorigenesis and metastasis. Oncogene. 2007;26(34):4951-60.

12. Wang Y, Sheng Q, Spillman MA, Behbakht K, Gu H. Gab2 regulates the migratory behaviors and E-cadherin expression via activation of the PI3K pathway in ovarian cancer cells. Oncogene. 2012;31(20):2512-20.

13. Horst B, Gruvberger-Saal SK, Hopkins BD, Bordone L, Yang Y, Chernoff KA, et al. Gab2-mediated signaling promotes melanoma metastasis. Am J Pathol. 2009:174(4):1524-33.

14. Ding C, Luo J, Yu W, Gao S, Yang L, Chen C, et al. Gab2 is a novel prognostic factor for colorectal cancer patients. Int J Clin Exp Pathol. 2015;8(3):2779-86

15. Liu X, Zhang Z, Sun L, Chai N, Tang S, Jin J, et al. MicroRNA-499-5p promotes cellular invasion and tumor metastasis in colorectal cancer by targeting FOXO4 and PDCD4. Carcinogenesis. 2011:32(12):1798-805.

16. Duranton B, Holl V, Schneider Y, Carnesecchi S, Gossé F, Raul F, et al. Polyamine metabolism in primary human colon adenocarcinoma cells (SW480) and their lymph node metastatic derivatives (SW620). Amino acids. 2003;24(1-2):63-72.

17. Zaravinos A. The Regulatory Role of MicroRNAs in EMT and Cancer. J Oncol. 2015:2015:865816.

18. Singh A, Settleman J. EMT, cancer stem cells and drug resistance: an emerging axis of evil in the war on cancer. Oncogene. 2010;29(34):4741-51.

19. Lamouille S, Xu J, Derynck R. Molecular mechanisms of epithelialmesenchymal transition. Nat Rev Mol Cell Biol. 2014;15(3):178-96.

20. Ma Y, Zhu B, Liu X, Yu H, Yong L, Liu X, et al. Inhibition of oleandrin on the proliferation show and invasion of osteosarcoma cells in vitro by suppressing Wnt/ß-catenin signaling pathway. J Exp Clin Cancer Res. 2015;34(1):115.

21. Smilek P, Dusek L, Veselý K, Rottenberg J, Kostrica R. Correlation of expression of Ki-67, EGFR, c-erbB-2, MMP-9, p53, bcl-2, CD34 and cell cycle analysis with survival in head and neck squamous cell cancer. J Exp Clin Cancer Res. 2006;25(4):549-55.

22. Zhang $X$, Min J, Wang Y, Li Y, Li H, Liu Q, et al. RABEX-5 plays an oncogenic role in breast cancer by activating MMP-9 pathway. J Exp Clin Cancer Res. 2013;32(1):52.

23. Buhmeida A, Bendardaf R, Hilska M, Collan Y, Laato M, Syrjänen S, et al. Prognostic significance of matrix metalloproteinase-9 (MMP-9) in stage ॥ colorectal carcinoma. J Gastrointest Cancer. 2009:40(3-4):91-7.

24. Jiang Y, Meng Q, Qi J, Shen H, Sun S. MiR-497 promotes metastasis of colorectal cancer cells through Nrdp1 inhibition. Tumour Biol. 2015;36(10):7641-7.

25. Liu F, Zhang T, Zou S, Jiang B, Hua D. B7H3 promotes cell migration and invasion through the Jak2/Stat3/MMP9 signaling pathway in colorectal cancer. Mol Med Rep. 2015;12(4):5455-60.

26. Worthley DL, Leggett BA. Colorectal cancer: molecular features and clinical opportunities. Clin Biochem Rev. 2010;31(2):31-8.

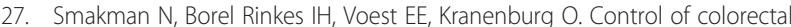
metastasis formation by K-Ras. Biochim Biophys Acta. 2005;1756(2):103-14. 
28. Giordano G, Febbraro A, Tomaselli E, Sarnicola ML, Parcesepe P, Parente D, et al. Cancer-related CD15/FUT4 overexpression decreases benefit to agents targeting EGFR or VEGF acting as a novel RAF-MEK-ERK kinase downstream regulator in metastatic colorectal cancer. J Exp Clin Cancer Res. 2015:34:108.

29. Lemieux E, Bergeron S, Durand V, Asselin C, Saucier C, Rivard N.

Constitutively active MEK1 is sufficient to induce epithelial-to-mesenchymal transition in intestinal epithelial cells and to promote tumor invasion and metastasis. Int J Cancer. 2009;125(7):1575-86.

30. Voisin L, Julien C, Duhamel S, Gopalbhai K, Claveau I, Saba-El-Leil MK, et al. Activation of MEK1 or MEK2 isoform is sufficient to fully transform intestinal epithelial cells and induce the formation of metastatic tumors. BMC Cancer. 2008;8:337.

31. Mira A, Isella C, Renzulli T, Cantarella D, Martelli ML, Medico E. The GAB2 signaling scaffold promotes anchorage independence and drives a transcriptional response associated with metastatic progression of breast cancer. Oncogene. 2009;28(50):4444-55.

32. Bentires-Alj M, Gil SG, Chan R, Wang ZC, Wang Y, Imanaka N, et al. A role for the scaffolding adapter GAB2 in breast cancer. Nat Med. 2006;12(1):114-21.

33. Herrera Abreu MT, Hughes WE, Mele K, Lyons RJ, Rickwood D, Browne BC, et al. Gab2 regulates cytoskeletal organization and migration of mammary epithelial cells by modulating RhoA activation. Mol Biol Cell. 2011;22(1):105-16.

34. Sun L, Zhang B, Liu Y, Shi L, Li H, Lu S. MiR125a-5p acting as a novel Gab2 suppressor inhibits invasion of glioma. Mol Carcinog. 2015. doi:10.1002/mc 22256.

35. Tan WH, Popel AS, Mac Gabhann F. Computational Model of Gab1/2Dependent VEGFR2 Pathway to Akt Activation. PLoS One. 2013;8(6):e67438.

\section{Submit your next manuscript to BioMed Central} and we will help you at every step:

- We accept pre-submission inquiries

- Our selector tool helps you to find the most relevant journal

- We provide round the clock customer support

- Convenient online submission

- Thorough peer review

- Inclusion in PubMed and all major indexing services

- Maximum visibility for your research

Submit your manuscript at www.biomedcentral.com/submit
Biomed Central 